\title{
From their Environment to their Behavior: A Procedural Approach to Model Groups of Virtual Agents
}

\author{
Rafael Hocevar ${ }^{1}$, Fernando Marson ${ }^{1}$, Vinícius Cassol $^{1}$, Henry Braun $^{1}$, \\ Rafael Bidarra ${ }^{2}$, and Soraia Musse ${ }^{1}$ \\ 1 Graduate Course in Computer Science \\ Pontifícia Universidade Católica do Rio Grande do Sul - Porto Alegre, Brazil \\ rafael.moura@acad.pucrs.br, soraia.musse@pucrs.br \\ ${ }^{2}$ Computer Graphics Group of Delft University of Technology - Delft, The Netherlands \\ R.Bidarra@tudelft.nI
}

\begin{abstract}
Simulation of everyday situations from real life can be a very useful tool in entertainment applications and training systems. Such applications, as games or computer animated movies usually need to provide virtual environments populated with virtual autonomous agents. Commonly, the agents need to be able to evolve in their environment, avoiding collision with each other and obstacles, besides interacting with other characters in order to provide realistic simulations. We present a model to simulate coherent group behaviors based on procedural modeling and semantic environments. Our main focus is virtual environments and agents, present in the background of games or movies generated with few/without user intervention.
\end{abstract}

\section{Introduction}

Human beings usually group with others in regular situations. When grouped, individuals interact with others according to their type of relationship, as well as the environment characteristics. Studies about human behaviors are produced since the 20 century $[1,2]$. The goal of such studies is to identify, for example, the distribution of individuals participation in small groups and also to analyze their interactions. Simulations of virtual agents interacting with others in a virtual environment can be applied in different areas, such as entertainment, engineering and security. We consider the method by [3], inspired in a biological algorithm, based on competition for space in a coherent growth of veins and branches [4]. Our main contributions are: i) to provide a strong connection between groups of virtual agents and the environment, i.e. world can be used to change the group behaviors; and ii) to enable group behaviors with fewer user interventions (agents characteristics are created as a function of environment and time). Simulations automatically generated using our technique allow to the animator be focused in the big picture and in the first plan characters [5]. Our model can be applied in games in order to coherently populate the environment (e.g. empty buildings). 


\section{Related Work}

Several aspects of group behaviors have been analyzed in the last years. Results of behavior groups analysis provide an useful reference for simulation/animation of groups and crowds [6-10]. Two important aspects that guide the motion of real people are: goal seeking, which reflect the target destination of each individual; and the least-effort strategy, reflecting the tendency of people to reach the goal along a path requiring the least effort [10]. Edward Hall [11] proposes the proxemics concept, which is the study of measurable distances between people as they interact. The specification of such distance can be based on different parameters: the agents relationship, the environment, the density of characters, among others. More specifically, concerning the motion of groups, Kamphuis and Overmars [12] introduce a two-phase approach, where a path for a single agent is generated by any motion planner. Then, a corridor is defined around the path, where all agents stay inside. Musse et al. [13] describes a model for controlling groups motion based on automatic tracking algorithms.

Recent works aim to produce coherently and realistically group behaviors taking into account steering and formation of groups. Karamouzas et al. [14] present a model where the velocity space to plan the avoidance maneuvers of each group is used to maintain a configuration that facilitates the social interactions between the group members. To provide the groups formation, the authors were inspired in the work developed by Moussaid et al. [15]. Such research shows that the majority of the pedestrians walks in small groups of up to three members following formations as Side by Side, V-Like and River-Like formations. In comparison, our groups also keep formation, however we firstly change group formation based on environment restrictions, and if there is free space, we also keep formation based on best efficiency in social behavior that is achieved in Side by Side formation [15]. An important contribution from our model is the connection between the population and the semantic environment, which constraints the motion behavior. Next section details our model.

\section{The Model}

Our model is mainly focused on the groups behaviors when evolving in a virtual environment regarding other groups location, density of agents in the space and environment characteristics (obstacles, interest locations, etc). It is important to emphasize that our model is suitable for background actors and actions, requiring minimum intervention of designers or users.

\subsection{Semantic Environment}

A Semantic Virtual Environment (SVE) $[16,17]$ is a virtual environment that is populated with entities enriched with semantics. A simulation environment is a complex space that is composed by a hierarchical set of simpler spaces, such as a city. Commonly, several neighborhoods composes a city, which are composed by many lots. These lots might have several types of buildings, with different types of rooms: kitchen, bedroom, bathroom, living room, among others. To specify goals in the environment 
we can assign a special attribute to any object that indicates some interesting thing or a resource that a certain object can provide. For instance, a TV provides fun as a chair provides rest. If an object that provides some resource is placed inside of a space, this space will provide that resource as well.

Our generator uses the Semantic Engine based on [18] in order to specify, create and store all spaces and objects. The specifications are made by a template file containing the spaces hierarchy and all objects that are instantiated in the environment. Once defined the environment, the next step is to create information about how to populate these spaces and what agents can do during the simulation. For that, we create a Population Class $(P C)$, which can automatically create a random population for a specific environment. As output of our SVE Generator we have a 2D layout that contains goals, walkable and non-walkable regions and a graph that will be used by the virtual agents to compute their paths. Another output is a 3D scene, that contains all $3 \mathrm{D}$ geometric representation of objects and spaces.

\subsection{Virtual Population}

This section describes how agents are generated in the VE, considering the $P C$ that is composed by following information: i) the simulation total time; ii) the higher density of agents to be attained during the simulation and the time it should occur. In this case, the simulation process is responsible for creating and destroying the agents (e.g. at the beggining and ending of a party) in order to attain the expected density of agents at a specific period of the simulation. Definition iii) is concerned with groups distribution presented in a certain population; and iv) the distribution of interest, entry and spawn locations where agents should be created or go to. In order to avoid user intervention, our model can automatically generate a population $P$ based on such definitions. From first frame until the expected peak of density of the simulation, agents are linearly created in the environment. The spaces and the objects previously defined can be the goals that are randomly distributed to the agents in the simulation. Once an agent reaches its goal, it stays there for a random time until a new goal is randomly selected. Moreover, agents can group with others. On the other hand, one or more groups $G$ can also be created into a specific $P$, i.e. people into $P$ that should physically interact are pre-defined, being maximum of 3 agents in each group, since mostly groups in real life are formed by up to 3 people [19]. When the distance between 2 members of $G_{i}$ is into a range defined by Hall's social distance [11], one group $G$ is formed. According to [3], the personal space for each agent $A$ is modeled as a circular region, that represents a "perception field" which can be used by each agent to avoid collision with others. In our case, we adopt another circular region we called group space (see Figure 1) which includes the $N$ members of $G_{i}$, and radius $R g$, computed based on follow Equations:

$$
\begin{aligned}
& A f=\operatorname{argmax}\left(\operatorname{dist}\left(A_{i}, \vec{C}\right)\right), \\
& R g=\operatorname{dist}\left(A_{A f}, \vec{C}\right)+\overline{R_{A f}},
\end{aligned}
$$


where $\vec{C}$ is the centroid of all $A_{i}$ positions and it is also the center of $G_{i}$ with radius $R g$, and $A f$ is the index of the agent which position is farther from the centroid $\vec{C}$. When agents are grouped they have equivalent goals and speeds.

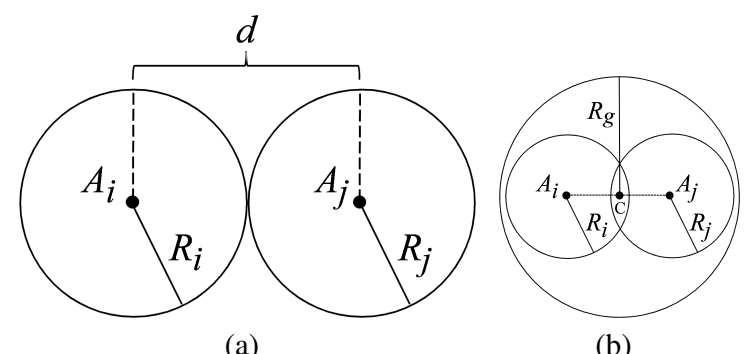

(a)

(b)

Fig. 1. a) Hall's [11] social distance (d) between two agents. b)If the agents are from same group $G$, a group space is defined representing the group as an unique entity.

\subsection{Simulation}

This phase is responsible for providing groups and agents motion and their interactions into the environment. Each group $G_{i}$ can evolve in a virtual environment, which can also be populated by other groups $G_{j}$. The following aspects are considered in order to define the individual behavior function of agent $A$ which is member of $G_{i}$ : i) agent's goal which is defined based on environment information; ii) density of agents close to $G_{i}$; iii) obstacles and other constraints around $G_{i}$; iv) location of close groups $G_{j}$. The agents movement is inspired in [20] algorithm. As in the original model, the environment is represented by a set of markers which discretizes the space. Overlayered to the markers, we create a grid of nodes in the space where motion is allowed and used as reference to the $A^{*}$ path planning algorithm, considering environment features [21, 22]. Basically, two group behaviors emerge from this connection between agents and environments. Firstly, grouped agents can present groups formation while evolving in the virtual environment. Secondly, they can vary their behavior (formation and trajectories) based on environment constraints and people density. In order to avoid that groups occupy the same space we keep a small region inside group space where the markers cannot be used for agents from a different group. After defined the members in a group, we are able to compute the physical agents position into the group space to determine the group formation. In such area, we can provide three formations, inspired in work proposed by [15] and illustrated in Figure 2.

The River-Like formation can be considered an emergent behavior in our model. Considering the agents with the same goal, being part of the same group, they are able to move in the same direction at the same speed, as in [20], emerging such formation. To provide the Side by Side formation, we perform a simple test of angles in order to keep the agents aligned and perpendicularly placed given their goal. At each agent step we 


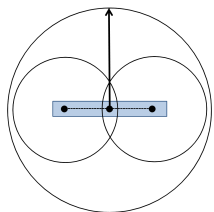

(a) Side by side.

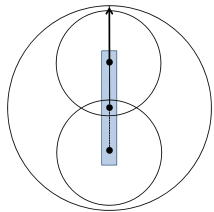

(b) River-like.

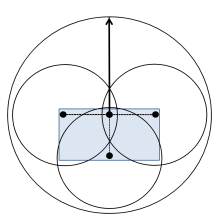

(c) V-formation.

Fig. 2. Group formations and the respectives ROIs.

check the angle between the vectors formed by the group centroid $\vec{C}$ to each agent and the vector from $\vec{C}$ to the next goal. When angle between these two vectors is greater than $90^{\circ}$ we increase the agent speed. For angles smaller than $90^{\circ}$, we decrease the agent speed. With such rules, the agents keep walking close to each other in a specific formation during the motion process (groups of 2 and 3 agents). Similar process is performed to obtain V-Formation, however, angles between agents and $\vec{C}$, as well as $\vec{C}$ and next goal should be approximately $45^{\circ}$. These formations are chosen as a function of the free space around the group. Our method considers the space in the goal direction trying to find out if there is available space for the group. Indeed, we compute for each group a 2D Region of Interest ( $R O I)$ that is included in group space and represents the minimum area that should exist in the environment for the group performance. These regions are simply computed based on agents positions and their sizes $A_{S}$. For this, we mathematically estimate the $R O I$ for the three group formations and test them against the environment space, computing $R O I_{\text {side }}, R O I_{V}$ and $R O I_{\text {river }}$. First test aims to keep the Side by Side formation, since it represents the best way, in social terms, for a group to go everywhere in low dense situations [15]. So, $R O I_{\text {side }}$ is checked if it can be included in the region around $G$. For instance, if $G$ is passing through a door with size smaller than $R O I_{\text {side }}$, then next group formation (V-Formation) is tested against the free space. If $R O I_{V}$ is still larger than the free space in the environment, then RiverLike formation is adopted.

\section{Results}

This section presents results obtained with our model (prototype implemented using Irrlicht Engine [23] and Cal3D [24]). Figure 3 represents a pool area in a backyard, where it is possible to observe modules of our model: on the left, it is illustrated the bounding boxes representing the semantic environment while on the right, a view of our simulation environment presents the agents and their paths provided by the path planning algorithm. We can observe a population (30 agents and 9 groups) automatic generated as well as their goals, initial locations and groups behaviors. Each group can be identified in Figure 3 by a circular area that represents the group space. We developed a framework to visualize the results of the simulation. The framework is also responsible for playing the animations accordingly the situation. Based on the agent's moving speed we are able to determine which animation should be played. Using data from the population, our algorithm is able to provide the agents motion across the environment. 
In such process, groups formations are performed by our agents (Side by Side, RiverLike, V-Formation). Moreover, the groups are able to identify the presence of other groups and compute a new path or new formation when needed. Figure 4 illustrates the visualization of a simulation example, where it is possible to observe the $3 \mathrm{D}$ virtual environment and also the virtual agents performing coherent group behaviors provided by our model.
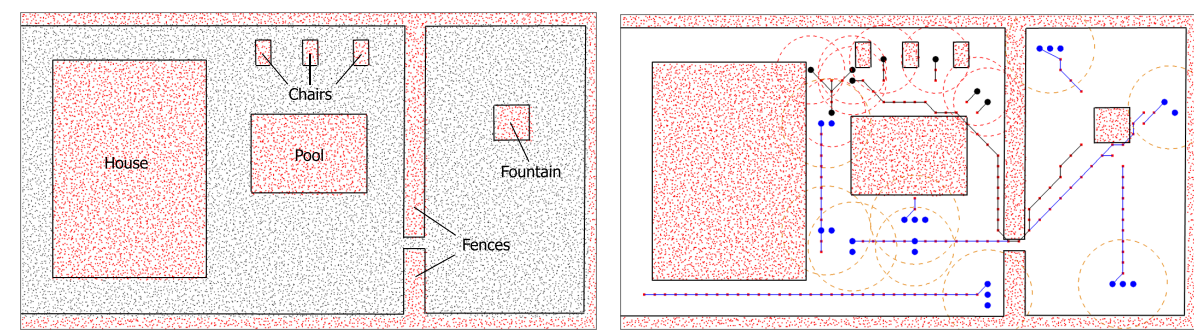

Fig. 3. On left, markers representing the walkable space. The dots inside boxes means regions where the motion in not allowed. On right, agents and their paths.
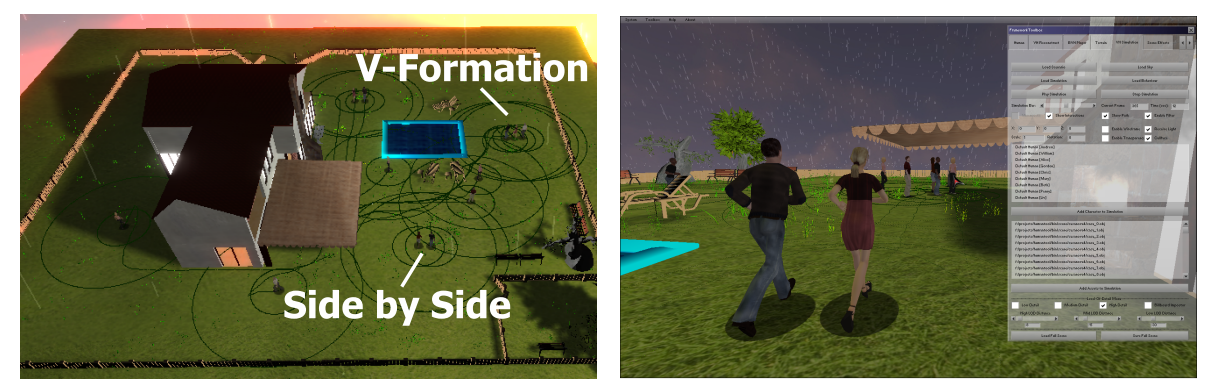

Fig. 4. Snapshots of our 3D framework, visualizing an example of simulation.

\section{Final Considerations and Future Works}

This paper presents a model to provide procedurally coherent group behaviors in a semantic 3D environment. Only the environment is manually created, while all agents and groups behaviors can be automatically generated. Each group is able to perform different behaviors (Side by Side, River-Like, V-Formation) while avoiding collision with other groups and objects in the space. The main goal is to generate semantic environment and behavior for background scenarios. Obtained results can be easily visualized in real-time with our 3D framework. As future work, we intend to provide virtual agents able to interact with objects. 


\section{Acknowledgments}

This work was supported by Brazilian research agencies CAPES, FAPERGS and FINEP using incentives of Brazilian Informatics Law ( $n^{\circ} 8.2 .48$ of 1991). The authors acknowledge the support granted by CNPQ and FAPESP to the INCT-SEC (National Institute of Science and Technology Embedded Critical Systems Brazil), processes 573963/2008-8 and 08/57870-9.

\section{References}

1. Chapple, E.D.: Quantitative analysis of the interaction of individuals. Proceedings of the National Academy of Sciences of the United States of America 25(2) (1939) pp. 58-67

2. Stephan, F.F., Mishler, E.G.: The distribution of participation in small groups: An exponential approximation. American Sociological Review 17(5) (1952) pp. 598-608

3. de Lima Bicho, A., Rodrigues, R.A., Musse, S.R., Jung, C.R., Paravisi, M., Magalhães, L.P.: Simulating crowds based on a space colonization algorithm. Computers \& Graphics 36(2) (2012) 70 - 79 Virtual Reality in Brazil 2011.

4. Sachs, T.: Polarity and the induction of organized vascular tissues. Annals of Botany 33(2) (1969) 263-275

5. Thalmann, D., Musse, S.R.: Crowd Simulation. Springer-Verlag London Ltd (2007)

6. Henderson, L.F.: The statistics of crowd fluids. Nature 229(5284) (1971) 381-383

7. Henderson, L.F.: On the fluid mechanic of human crowd motions. Transportation Research 8(6) (1974) 509-515

8. Fruin, J.J.: Pedestrian and planning design. Metropolitan Association of Urban Designers and Environmental Planners, Elevator World Inc., New York, NY, USA (1971)

9. Helbing, D.: Pedestrian dynamics and trail formation. In Schreckenberg, M., Wolf, D.E., eds.: Traffic and Granular Flow '97, Singapore, Springer-Verlag (1997) 21-36

10. Still, G.K.: Crowd Dynamics. PhD thesis, University of Warwick, Coventry, UK (2000)

11. Hall, E.T.: The hidden dimension / Edward T. Hall. [1st ed.] edn. Doubleday, Garden City, N.Y. : (1966)

12. Kamphuis, A., Overmars, M.H.: Finding paths for coherent groups using clearance. In: Proceedings of the ACM SIGGRAPH/Eurographics Symposium on Computer Animation, Aire-la-Ville, Switzerland, Switzerland, Eurographics Association (2004) 19-28

13. Musse, S.R., Jung, C.R., Jr, J.C.S.J., Braun, A.: Using computer vision to simulate the motion of virtual agents. Computer Animation and Virtual Worlds 18(2) (2007) 83-93

14. Karamouzas, I., Overmars, M.: Simulating the local behaviour of small pedestrian groups. In: Proceedings of the 17th ACM Symposium on Virtual Reality Software and Technology. VRST '10, New York, NY, USA, ACM (2010) 183-190

15. Moussaïd, M., Perozo, N., Garnier, S., Helbing, D., Theraulaz, G.: The walking behaviour of pedestrian social groups and its impact on crowd dynamics. PLoS ONE 5(4) (2010) e10047

16. Otto, K.A.: Semantic virtual environments. In: Special interest tracks and posters of the 14th international conference on World Wide Web, New York, NY, USA, ACM (2005) 1036-1037

17. Gutierrez, M., Vexo, F., Thalmann, D.: Semantics-based representation of virtual environments. International Journal of Computer Applications in Technology 23(2-4) (2005) 229-38 Virtual Reality Lab., Ecole Polytech. Fed. de Lausanne, Switzerland.

18. Kessing, J., Tutenel, T., Bidarra, R.: Services in game worlds: A semantic approach to improve object interaction. In: Proceedings of the 8th International Conference on Entertainment Computing. ICEC '09, Berlin, Heidelberg, Springer-Verlag (2009) 276-281 
19. Mills, T.M.: The sociology of small groups / Theodore M. Mills. Prentice-Hall, Englewood Cliffs, N.J. (1967)

20. Rodrigues, R.A., de Lima Bicho, A., Paravisi, M., Jung, C.R., Magalhaes, L.P., Musse, S.R.: An interactive model for steering behaviors of groups of characters. Appl. Artif. Intell. 24 (2010) 594-616

21. Hart, P.E., Nilsson, N.J., Raphael, B.: A formal basis for the heuristic determination of minimum cost paths. SIGART Bull. (1972) 28-29

22. Cassol, V.J., Marson, F.P., Vendramini, M., Paravisi, M., Bicho, A.L., Jung, C.R., Musse, S.R.: Simulation of autonomous agents using terrain reasoning. In: Proc. the Twelfth IASTED International Conference on Computer Graphics and Imaging (CGIM 2011), Innsbruck, Austria, IASTED/ACTA Press (2011)

23. Irrlicht Engine: http://irrlicht.sourceforge.net (2012)

24. Cal3D: 3d Character Animation Library: http://gna.org/projects/cal3d (2012) 